\title{
Stabilization of Lactobacillus sp. with enhanced thermal resistance by spray-drying
}

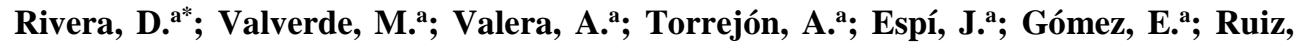
B. ${ }^{\mathrm{a}}$.

a AINIA Centro Tecnológico. Valencia, Spain

*E-mail of the corresponding author: jdrivera@ainia.es

\begin{abstract}
Combining biotechnology and encapsulation by spray-drying is possible to obtain microorganisms-based products in powder format without losing viability. A methodology based on the improvement of the thermal stress resistance of selected strains of Lactobacillus sp. and subsequent culture stabilization by spray-drying by optimizing the process parameters and using thermal protectant materials has been developed. The results obtained showed that the final product kept the viability of the initial culture in addition to be a solid, powdery and ease-to-handle product.
\end{abstract}

Keywords: thermal resistance; spray-drying; probiotics; viability; fermentation; encapsulation. 


\section{Introduction}

Sectors such as food and agriculture are looking for products based on microorganisms (i.e. probiotics, biofertilizers) with the aim of conferring a beneficial physiological effect on the host; most of them are available in liquid form. The challenge of these sectors is to offer them in powder formats for decreasing costs and to facilitate their use and applications. Vacuum and freeze drying are two drying technologies used for this end. The main disadvantages of freeze-drying are the high energy input and long processing times [1].Vacuum drying is faster and cheaper because it operates at a temperature above the freezing point. Other dryin technology, convective drying, can cost from 4 to 8 times less than freeze drying. However, the quality of the obtained dried products can be much lower, with a drastic reduction in the volume and colour changes [2].

Spray drying is a widely used technology to dry liquids and it is used in different sectors of food industry ensuring microbiological stability avoiding risks of chemical and/or biological degradations and obtaining product with specific properties like instantaneous solubility [3]. Processing costs are six times lower than freeze drying [2,4], however, the high temperature can lead to cell damage. The enhancement of the thermal resistance of the cell culture can be one alternative [5]. The addition to the medium of ingredients that may induce osmotic shock may strengthen the cell membrane [6]. The preadaptation of cells to heat is another possible strategy. During thermal stress, proteins heat shock is produced. These proteins may help to repair misfolded proteins during spray drying [6]. The use of protectant materials during the spray drying process can also enhance the survival of the microorganisms [7,8] being carbohydrates, such as trehalose, glucose, maltodextrin, inulin, fructo-oligosaccharides or potato starch [5], the most commonly used in food industries [9].

This microencapsulation processes can increase the microorganisms survival, not only during the spraying process, but also providing extra protection against ambient conditions or gastrointestinal conditions, allowing probiotics to reach the gut in an adequate amount $[10,11]$.

The aim of this study was to obtain microorganisms-based products in a powder format without losing viability by combining biotechnology and spray-drying. For this purpose, this study has been focused on different aspects: process variables, including formulation of the feed (thermal protectants, and encapsulating materials) and different stress methods for the culture.

\section{Materials and Methods}

\subsection{Materials}

A probiotic strain of Lactobacillus sp. isolated in previous works from cured meat was used in this study. The reference culture medium was MRS Broth, purchased from VWR. 
Osmotic stress was induced by bifferent amounts of Sodium Chloride (Scharlau). Different protectant materials were used in the spray drying tests; Maltodextrin from Roquette, Trehalose, from Hayashibara, and modified starch, from Ingredion. All of them were food grade products.

\subsection{Methods}

\subsubsection{Production of Lactobacillus sp. culture}

For the screening of the spray drying conditions, a single batch of $20 \mathrm{~L}$ of Lactobacillus sp was produced using a stirring tank bioreactor (BIOSTAT C plus from Sartorius Stedim, Germany). The operating conditions were: Temperature: $37^{\circ} \mathrm{C}$; Medium: MRS Broth 52 $\mathrm{g} \cdot \mathrm{L}^{-1}$. Dissolved Oxygen $>40 \%$ (controlled by cascade). When the culture reached the stationary phase, the culture was harvested using a high-speed refrigerated bench centrifuge (Sigma 6K15). After the harvesting, viable count of the product was done in order to know the concentration of the culture, measured in colony-forming units (CFU/mL).

\subsubsection{Stabilization of the culture}

The cultures of Lactobacillus sp. were stabilized by spray drying using a laboratory spray dryer (Büchi B-290, Switzerland). The operating conditions were: feed rate: 3-6 $\mathrm{ml} \cdot \cdot \mathrm{min}^{-1}$; inlet temperatures: $105-115^{\circ} \mathrm{C}$. Outlet temperatures between 51 and $71^{\circ} \mathrm{C}$. The tests were performed with different carriers: thermal protectant materials and encapsulating materials (Table 1).

Table 1. Concentration of the thermal protectant materials

\begin{tabular}{|c|c|c|c|}
\hline Samples & $\begin{array}{c}\text { Trehalose } \\
(\%)(\mathbf{m} / \mathbf{m})\end{array}$ & $\begin{array}{c}\text { Maltodextrin } \\
(\%)(\mathbf{m} / \mathbf{m})\end{array}$ & $\begin{array}{l}\text { Starch } \\
(\%)(\mathbf{m} / \mathbf{m})\end{array}$ \\
\hline $1-3$ & 30,20 & --- & --- \\
\hline $4-7$ & 7,50 & 23,26 & --- \\
\hline 8-11 & 7,50 & 15,53 & 7,76 \\
\hline $12-42$ & 4,28 & 9,15 & 4,57 \\
\hline
\end{tabular}

2.2.3. Preadaptation of Lactobacillus cultures to spray drying to enhance thermal resistance.

Shake flask cultures were performed to study the effect of the culture conditions in the final viability of the spray dried products. Two effects were studied (thermal and osmotic stress) and Surface Response Methodology (SRM) was used for the design of a 3-level factorial experimental design in 9-runs with triplicates. The following variables were selected as response: the concentration of the cultures $(\mathrm{CFU} / \mathrm{mL})$, the concentration of the microencapsulated microorganism (CFU/g) and the viability of the product after spray drying was calculated as follow: 


$$
\text { Viability }(\%)=\frac{\log \left(\text { Total } C F U_{\text {spray drying }}\right)}{\log \left(\text { Total } C F U_{\text {culture }}\right)}
$$

The cultures were grown in $500 \mathrm{~mL}$ shake flask with $150 \mathrm{~mL}$ of MRS broth. The initial optical density of the cultures, measured at $600 \mathrm{~nm}$, was adjusted to 0.1 The cultures were maintained at $37^{\circ} \mathrm{C}$ and $150 \mathrm{rpm}$. Different levels of thermal and osmotic stress were applied to the cultures according to the following experimental design.

To induce osmotic stress, when the cultures reached an optical density of $0,4-0,5$, they were centrifugated and resuspended to $150 \mathrm{~mL}$ of MRS or MRS broth supplemented with Sodium Chloride (according to Table 2). To induce thermal stress, when the cultures reached an optical density of 0,8-1,0, the flasks were introduced in a water bath at the specified temperature (according to Table 2). The cultures were spray-dried once they reached stationary phase (30 hours of process).

Table 2. Experimental Design.

\begin{tabular}{lcc}
\hline & Thermal Stress $\left({ }^{\mathbf{0}} \mathbf{C}\right)$ & Osmotic Stress (M) \\
\hline R-1 & 49 & -- \\
R-2 & 42 & 0,3 \\
R-3 & 49 & 0,6 \\
R-4 & -- & 0,3 \\
R-5 & 42 & 0,6 \\
R-6 & 42 & -- \\
R-7 & 49 & 0,3 \\
R-8 & -- & -- \\
R-9 & -- & 0,6 \\
\hline
\end{tabular}

\section{Results}

\subsection{Effect of the inlet temperature}

First of all, several tests of Lactobacillus sp. culture were carried out with the aim to know the effect of the inlet temperature in the microorganisms viability. In these tests, trehalose was used as material for thermal protection.

Table 3. Effect of the inlet temperature on the microorganisms viability

\begin{tabular}{lccc}
\hline Sample & Tin $\left({ }^{\circ} \mathbf{C}\right)$ & Tout $\left({ }^{\circ} \mathbf{C}\right)$ & Viability (\%) \\
\hline 1 & 105 & 55 & $79,6 \%$ \\
2 & 110 & 68 & $75,6 \%$ \\
3 & 115 & 71 & $80,7 \%$ \\
\hline
\end{tabular}

As expected, low inlet temperatures give, in general terms, high viability. Opposite to what it might appear, inlet temperature do not have a direct effect on the survival of microorganisms. The reason is that the viability does not depend only on the initial temperature, but also on the residence time and, in general, the temperature profile of the process, from the feed to the final dried product. In this situation, the outlet temperature has 
a remarkable importance. The residence time depends on several variables of the process, feed composition and concentration, and also de design of the spray dryer equipment.

\section{Effect of the feed composition}

Table 4 shows the feed composition and the results of viability. Trials 12 to 15 were performed using the same carriers but with a lower concentration, as shown in Table 1.

Fig. 1 shows the effect of the feed composition and the inlet temperature, on the microorganisms viability. It can be seen that the effect of the feed composition, specially its concentration, are the relevant aspects to increase the viability in the final product.

Best results of viability were achieved for an inlet temperature of $115^{\circ} \mathrm{C}$ combined with the trehalose/maltodextrin/starch as protectants. As shown in Fig. 2, the feed composition (Carriers) is the most relevant parameter on the microorganisms viability in the final product. The effect of the feed composition is bigger than the temperature due to its high estadistical significance. The selection of the carrier is relevant, but its concentration in the feed is even more relevant. This effect is due to that a lower concentration implies lower outlet temperatures and therefore, higher viability. However, lower carrier concentration in the feed leads to bigger costs, so this parameter needs to be optimized for industrial processes.

Table 4. Effect of the thermal protectants and wall materials on the microorganisms viability

\begin{tabular}{lccc}
\hline Sample & Feed Composition & $\begin{array}{c}\text { Inlet } \mathbf{T} \\
\left({ }^{\mathbf{}} \mathbf{C}\right) \mathbf{( \pm \mathbf { 2 } ^ { \mathbf { } } \mathbf { C } )}\end{array}$ & $\begin{array}{c}\text { Viability } \\
\mathbf{( \% )}\end{array}$ \\
\hline 1 & Trehalose & 105 & $79,6 \%$ \\
2 & Trehalose & 110 & $75,6 \%$ \\
3 & Trehalose & 115 & $80,7 \%$ \\
4 & Trehalose/maltodextrin & 100 & $81,5 \%$ \\
5 & Trehalose/maltodextrin & 105 & $80,3 \%$ \\
6 & Trehalose/maltodextrin & 110 & $79,3 \%$ \\
7 & Trehalose/maltodextrin & 115 & $77,0 \%$ \\
8 & Trehalose/maltodextrin/Starch & 100 & $77,1 \%$ \\
9 & Trehalose/maltodextrin/Starch & 105 & $78,8 \%$ \\
10 & Trehalose/maltodextrin/Starch & 110 & $81,6 \%$ \\
11 & Trehalose/maltodextrin/Starch & 115 & $75,0 \%$ \\
12 & Trehalose/maltodextrin/Starch & 100 & $78,3 \%$ \\
13 & Trehalose/maltodextrin/Starch & 105 & $82,4 \%$ \\
14 & Trehalose/maltodextrin/Starch & 110 & $87,3 \%$ \\
15 & Trehalose/maltodextrin/Starch & 115 & $87,9 \%$ \\
\hline
\end{tabular}




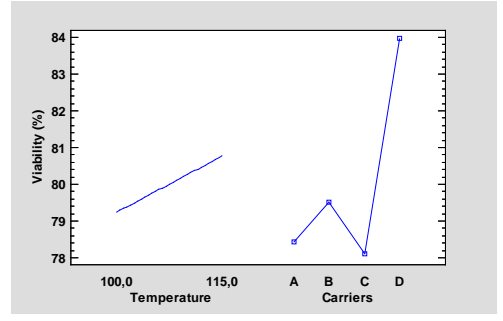

Fig. 1 Principal effects for viability (\%)

A: Trehalose (Sample 1-3)

B:Trehalose/maltodextrin (Sample 4-7)

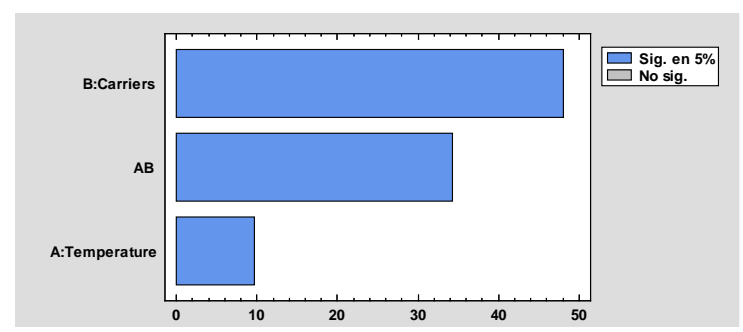

Fig. 2 Pareto Diagram for $\mathrm{CFU/g}$

C: Trehalose/maltodextrin/Starch (sample 8-11)

D: Trehalose/Maltodextrin/Starch (Sample 12-15)

Trehalose with maltodextrin seems to produce slightly better results than the combination of three carriers, but the addition of starch improves the encapsulation effect on the particles. The encapsulation of the microorganisms, lead to improved protection, longer shelf life, and lower damage during gastrointestinal tract in case of probiotics.

\subsection{Preadaptation of Lactobacillus cultures to spray drying to enhance thermal resistance.}

The average results of the response variables selected for the experimental design on Table 2 are shown in Table 5. Taking into consideration the previous results of viability, mass yield, and the effect of the inlet temperature;this last was fixed at $105^{\circ} \mathrm{C}$ for the rest of the study. As it can be shown, the variations in the fermentative process did not affect significantly the viable count of the culture. However, they had an effect in the viable count of the microencapsulated product and therefore in the final viabilitiy.

Table 5. Preadaptation of Lactobacillus cultures to spray drying to enhance thermal resistan

\begin{tabular}{|c|c|c|c|c|c|}
\hline & $\begin{array}{c}\text { Thermal } \\
\text { Stress }\left({ }^{\circ} \mathrm{C}\right) \\
\left( \pm 2^{\circ} \mathrm{C}\right)\end{array}$ & $\begin{array}{c}\text { Osmotic } \\
\text { Stress (M) } \\
( \pm 0,01 M)\end{array}$ & $\mathrm{CFU} / \mathrm{mL}$ & CFU/g & Viability (\%) \\
\hline $\mathrm{R}-1$ & 49 & -- & $2,66 \cdot 10^{9}$ & $2,67 \cdot 10^{8}$ & $88,57 \%$ \\
\hline $\mathrm{R}-2$ & 42 & 0,3 & $4,27 \cdot 10^{9}$ & $2,12 \cdot 10^{9}$ & $95,11 \%$ \\
\hline $\mathrm{R}-3$ & 49 & 0,6 & $2,16 \cdot 10^{9}$ & $1,00 \cdot 10^{9}$ & $94,61 \%$ \\
\hline $\mathrm{R}-4$ & -- & 0,3 & $3,42 \cdot 10^{9}$ & $2,97 \cdot 10^{8}$ & $88,40 \%$ \\
\hline $\mathrm{R}-5$ & 42 & 0,6 & $2,63 \cdot 10^{9}$ & $1,40 \cdot 10^{9}$ & $95,24 \%$ \\
\hline R-6 & 42 & -- & $2,23 \cdot 10^{9}$ & $2,75 \cdot 10^{8}$ & $89,59 \%$ \\
\hline $\mathrm{R}-7$ & 49 & 0,3 & $5,85 \cdot 10^{9}$ & $1,25 \cdot 10^{8}$ & $84,07 \%$ \\
\hline $\mathrm{R}-8$ & -- & -- & $3,58 \cdot 10^{9}$ & $3,08 \cdot 10^{8}$ & $88,63 \%$ \\
\hline $\mathrm{R}-9$ & -- & 0,6 & $2,64 \cdot 10^{9}$ & $1,45 \cdot 10^{9}$ & $94,89 \%$ \\
\hline
\end{tabular}




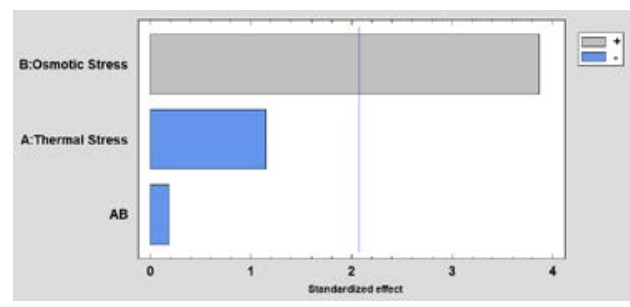

Fig. 3 Pareto chart of standardized effects for sprayed product viable count, Log (CFU/g)

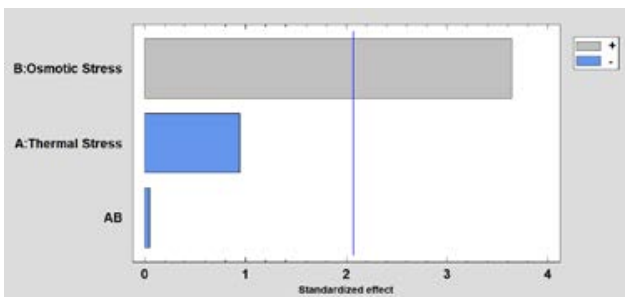

Fig. 4 Pareto chart of standardized effects for sprayed product viability (\%)

According to Fig. 3, Osmotic stress has a significative effect in the final viability (\%) as well in the final concentration of the microorganisms (CFU/g).The optimal conditions for maximizing the final viable count and viability are shown in Fig. 5, Fig. 6, Fig. 7 and Fig. 8.

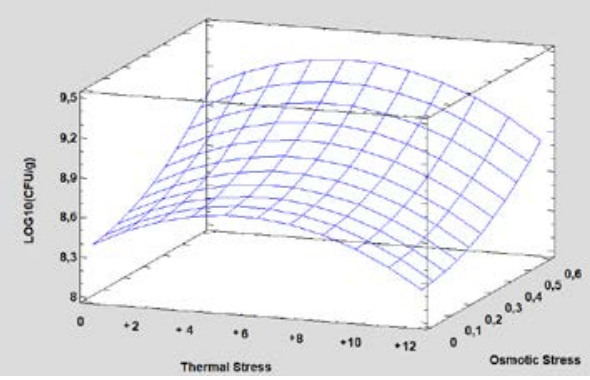

Fig. 5 Estimated surface response for sprayed product viable count, $\mathrm{Log}$ (CFU/g)

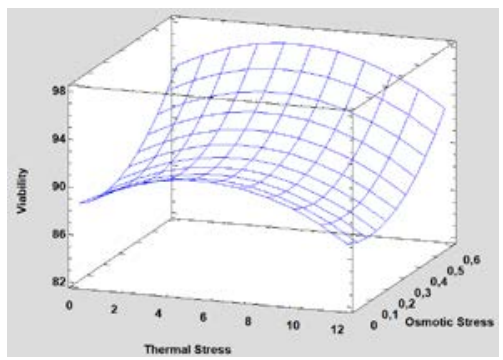

Fig. 7 Estimated surface response for sprayed product viability

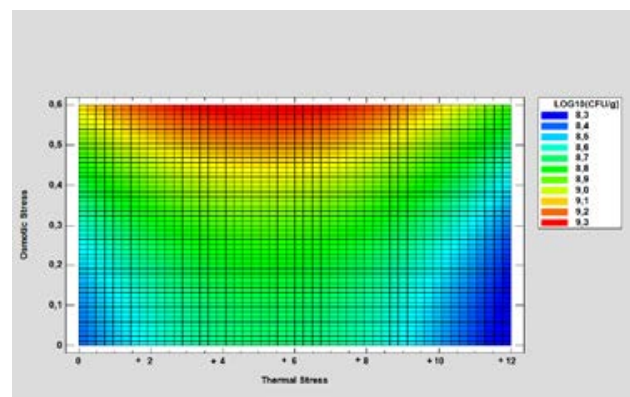

Fig. 6 Contours for estimated response surface for sprayed product viable count, $\log (\mathrm{CFU} / \mathrm{g})$

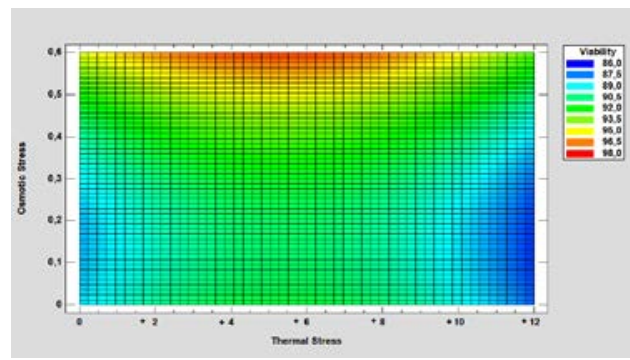

Fig. 8 Contours for estimated response surface for sprayed product viability

For the final viability of the product, the estimated surface response shows that the optimal value for the viability, which can reach $97,264 \%$ could be achieved by applying osmotic stress of $0,6 \mathrm{M}$ and a thermal shock at $43,28^{\circ} \mathrm{C}$. 


\section{Conclusions}

Thermal processing of microorganisms for their stabilization and encapsulation can be done by Spray drying without loosing viability. Combining biotechnology and encapsulation by spray-drying is possible to obtain microorganisms-based products in powder format keeping their viability.

Feed composition has a highlighted effect on viability. Some carries as trehalose can be included as thermal protectants, improving microorganisns resistance against temperature. Other carriers, can be included with the objective of improving process yields, or giving added valuable properties to the final product, as encapsulating materials.

The concentration of carriers of the feed has also a remarkable effect, because lower concentrations lead to lower temperatures during the process. However, it is directly related to processes costs, so it must be optimized for industrial processes.

In addition, modificantions in the fermentative process of the microorganism, as a preadaptatiton of the cells to spray drying may increase even more the final viability of the product. According to the results of the present study, the final viability of the product may increase from $79,6 \%$ to $82,4 \%$ by the apropiate selection of protective materials, and from $82,4 \%$ to $95,2 \%$ by the preadaptation of the microorganism during its fermentation.

\section{References}

[1] Nedovic, V.; Kalusevic, A.; Manojlovic, V.; Levic, S.; Bugarski, B. An overview of encapsulation technologies for food applications. Procedia Food Science 2011, 1, 1806-1815.

[2] Barbosa, J.; Borges, S.; Amorinm, M.; Pereira, M.J.; Oliveira, A.; Pintado, M.E.; Teixeira, P. Comparison of spray drying, freeze drying and convective hot air drying for the production of a probiotic orange powder. Journal of Functional Foods 2015, 17, 340-351.

[3] Gharsallaoui, A.; Roudaut, G.; Chambin, O.; Voilley, A.; Saurel, R. Applications of spray-drying in microencapsulation of food ingredients: An overview. Food Research International 2007, 40, 1107-1121.

[4] Knorr, D. Technology aspects related to microorganisms in functional foods. Trends in Food Science \& Technology 1998, 9, 295-306.

[5] Huang, S.; Vignolles, M.-L.; Chen, X.D.; Loir, Y.L.; Jan, G.; Schuck, P.; Jeantet, R. Spray drying of probiotics and other food-grade bacteria: A review. Trends in Food Science \& Technology 2017, 63, 1-17.

[6] Fu, N; Chen, X. D. Towards a maximal cell survival in convective thermal drying processes. Food Research International 2011, 44, 1127-1149.

[7] Liu, H.; Gong, J.; Chabot, D.; Miller, S.S.; Cui, S.W.; Ma, J.; Zhong, F.; Wang, Q. Protection of heatsensitive probiotic bacteria during spray-drying by sodium caseinate stabilized fat particles. Food Hydrocolloids 2015, 51, 45-467.

[8] Arslan, S.; Erbas, M.; Tontul, I.; Topuz, A. Microencapsulation of probiotic Saccharomyces cerevisiae var. boulardii with different wall materials by spray drying. LWT - Food Science and Technology 2015, 63, 685-690.

[9] Santivarangkna, C.; Higl, B.; Foerst, P. Protection mechanisms of sugars during different stages of preparation process of dried lactic acid starter cultures. Food Microbiology 2008, 25, 429-441.

[10] Li XY, Chen XG, Sun ZW, Park HJ, Cha D-S. 2011. Preparation of alginate/chitosan/ carboxymethyl chitosan complex microcapsules and application in Lactobacillus casei ATCC 393. Carbohydr Polym 83:1479e85

[11] Desai KGH, Park HJ. 2005. Recent developments in microencapsulation of food ingredients. Drying Technol 23:1361e94. 